\title{
Statistical mechanics of nucleation: Incorporating translational and rotational free energy into thermodynamics of a microdroplet
}

\author{
Isamu Kusaka \\ The Koffolt Laboratories, The Department of Chemical and Biomolecular Engineering, The Ohio State University, \\ 140 West 19th Avenue, Columbus, Ohio 43210, USA \\ (Received 26 July 2005; published 23 March 2006)
}

\begin{abstract}
Motivated by the original Lothe-Pound prescription, we derive a formula for the replacement partition function that is suitable for vapor to liquid phase nucleation in terms of the classical phase integral. The resulting expression was evaluated for truncated and shifted Lennard-Jones fluids by means of computer simulation. The corresponding Lothe-Pound correction factor, which is to multiply the classical expression for the nucleation rate, decreases significantly with increasing temperature and decreasing cluster size, and is in the range of $10^{9}-10^{13}$ for the temperatures and the cluster sizes we studied. This is somewhat smaller than the original estimate of $10^{17}$ by Lothe and Pound.
\end{abstract}

DOI: 10.1103/PhysRevE.73.031607

PACS number(s): 82.60.Nh, 64.60.Qb, 68.55.Ac

\section{INTRODUCTION}

In a theory of vapor to liquid nucleation, the equilibrium cluster size distribution $c_{n}$, i.e., the number density of clusters each consisting of $n$ molecules, plays an important role. In principle, the framework of statistical mechanics can be used to predict $c_{n}$ in terms of the partition function of the $n$-sized cluster. In classical nucleation theory, $c_{n}$ is related to the reversible work required to form the cluster inside the supersaturated vapor, which in turn is expressed in terms of macroscopic thermodynamic quantities such as the chemical potentials and the surface tension.

In 1962, Lothe and Pound pointed out that a proper account of the translational and the rotational free energies is missing in this classical approach, and argued that the classical theory prediction of $c_{n}$ must be multiplied by a correction factor as large as $10^{17}$ [1]. Though there is now a consensus in the field of nucleation in regard to the necessity of some correction factor, a factor this large appears excessively large to many, and in some cases, destroys a fairly good agreement with experimental findings. Thus, the claim of Lothe and Pound has generated serious controversy as summarized in Refs. [2-8]. For example, Reiss and his coworkers arrived at a correction factor in the range of $10^{3}-10^{6}$ [9-12]. This discrepancy, as we shall see, results from different interpretations given in these theories to the classical theory expression for the reversible work. One might expect that a discrepancy of this magnitude allows for a satisfactory resolution of the disagreement by means of experiments. However, there is also a considerable uncertainty in the value of the surface tension of a microscopic cluster [13-16]. Thus, either theory could be brought to agreement with experiments if some reasonable change is made to the value of the surface tension. Consequently, an accurate evaluation of the correction factor must still be regarded as an open question.

The problem we are concerned with here, of course, does not arise in a purely statistical mechanical approach to nucleation. Despite considerable progress in both our understanding of statistical mechanics of nucleation and computational techniques in recent years [17-22], however, classical theory remains the most useful theory in correlating the outcomes of nucleation experiments. Moreover, a similar problem is believed to the arise in the statistical mechanical density functional theory approach to nucleation [23-26]. Thus, it appears worthwhile to reconsider the issue closely.

Throughout the development of the Lothe-Pound theory, the correction factor was estimated using models more suitable for vapor to crystal phase nucleation [27-35]. More than 40 years after the seminal work of Lothe and Pound, however, we have yet to evaluate in any direct fashion the numerical value of the correction factor suitable for vapor to liquid phase nucleation based on the original Lothe-Pound prescription. In fact, there exists no mathematical expression of the quantity in this case on the basis of which such a numerical evaluation can be envisioned. To a large extent this is due to a lack of detailed knowledge of liquid structure and sufficiently powerful computational tools at the time when the validity of the theory was most intensely debated. Building on the original Lothe-Pound prescription [27], we shall thus derive a formula for the correction factor suitable for the vapor to liquid phase nucleation and evaluate it by means of computer simulation of the bulk liquid.

\section{THE LOTHE-POUND THEORY}

According to a statistical mechanical formulation [8-11], the equilibrium cluster size distribution $c_{n}$ in a metastable pure vapor is given by

$$
c_{n}=\frac{q_{n}}{V} e^{\mu_{v} n / k_{B} T}
$$

where $k_{B}$ is Boltzmann's constant, $T$ is the absolute temperature of the system, $V$ is the system volume, and $\mu_{v}$ is the chemical potential of the vapor phase at $T$ and pressure $p_{v}$. Limiting our attention to classical mechanical systems, we write the partition function $q_{n}$ of the $n$-sized cluster as 


$$
q_{n}=\frac{1}{\Lambda^{3 n} n !} \int_{\mathbf{R}_{c, m} \in V}^{\prime} d \mathbf{r}^{n} e^{-U_{n} / k_{B} T},
$$

where $\mathbf{r}^{n}$ collectively denotes the coordinates of $n$ particles, $U_{n}$ is their interaction potential, and $\Lambda$ is the thermal wavelength of a particle. The integral is to be taken under the constraint, as denoted by the prime, that the $n$ particles form a cluster, whatever its definition might be. The position of the center of mass $\mathbf{R}_{c . m}$. of this cluster must be confined to $V$, a condition denoted here by $\mathbf{R}_{c . m} \in V$. We note that $V$ can be taken arbitrarily large compared to the physical dimension of the cluster, and hence $q_{n}$ is proportional to $V$. It is convenient to rewrite Eq. (1) as

$$
c_{n}=z_{n} e^{\mu_{v} n / k_{B} T},
$$

where $z_{n}=q_{n} / V$. From a standard relation in statistical mechanics,

$$
e^{-\mu_{v} n / k_{B} T}=\left[Y\left(T, p_{v}, N\right)\right]^{n / N},
$$

in which $Y$ is the isothermal-isobaric partition function of a vapor phase consisting of some macroscopic number $N$ of particles and held at constant $T$ and $p_{v}$. In what follows, we shall write $Y_{v}$ for the value of $Y$ evaluated for the vapor phase at $\left(T, p_{v}, N\right)$.

In an attempt to relate $z_{n}$ to macroscopically measurable quantities, one first defines $W$ by the equation

$$
c_{n}=\frac{N_{1}}{V} e^{-W / k_{B} T},
$$

where $N_{1}$ is the number of monomers in the system. Combining Eqs. (3)-(5), we obtain

$$
W=-k_{B} T \ln \frac{\left(V / N_{1}\right) z_{n}}{Y_{v}^{n / N}},
$$

whose physical content can be made more transparent by rewriting it as

$$
W=-k_{B} T \ln \frac{\left(V / N_{1}\right) z_{n} Y_{v}^{(N-n) / N}}{Y_{v}} .
$$

If the interaction potential between the cluster and the surrounding vapor is negligible, as is assumed in arriving at Eq. (1), the quantity $W$ is identified with the reversible work required to convert $n$ of the vapor particles into an $n$-sized cluster, which has a free energy contribution from the free translation within the volume $V / N_{1}$ and the free rotation. Provided that the vapor phase can be treated as an ideal gas and that $N \approx N_{1}$, it is straightforward to verify that $W=0$ for $n=1$ and for a sufficiently large value of $N$.

In classical nucleation theory, one estimates $W$ based on the following expression obtained by Gibbs [36] for the reversible work required to form a critical nucleus, which by definition is in equilibrium with the surrounding vapor:

$$
W_{G}=-V_{s}\left(p_{l}-p_{v}\right)+\sigma A_{s},
$$

where $p_{l}$ is the pressure of the reference bulk liquid having the same temperature and chemical potential as the vapor. $A_{s}$ is the area of the surface of tension, which is assumed to be spherical, and $V_{s}$ is the volume enclosed by this surface. The surface tension between the metastable vapor phase and the nucleating liquid phase is denoted by $\sigma$. We emphasize that $\sigma$ pertains to a critical nucleus, and hence differs from the surface tension $\sigma_{\infty}$ measured for a flat interface separating the vapor and liquid phases at phase coexistence. Since $\sigma$ is not accessible to experiment, one typically uses $\sigma_{\infty}$ in place of $\sigma$. We shall avoid making this substitution as it is unnecessary in the conceptual development of the theory that follows. Strictly speaking, Eq. (8) is valid only for a critical nucleus, and an additional term arises when the Gibbs theory of interface is properly extended to treat nonequilibrium clusters [37-41]. We will not be concerned with this aspect of classical theory here. In any event, classical theory thus claims that

$$
W_{G}=-k_{B} T \ln \frac{\left(V / N_{1}\right) z_{n}}{Y_{v}^{n / N}} .
$$

It is straightforward to show that, if $W_{G}$ ever becomes zero, then $\sigma, V_{s}$, and hence $A_{s}$ must vanish simultaneously [36]. It is quite reasonable to expect that $W_{G}$ indeed vanishes at the onset of the mean-field spinodal where the system becomes unstable with respect to any infinitesimal fluctuation in density. This implies that the vapor phase, even if it is constrained to be homogeneous at a microscopic level, already contains critical nuclei. Thus, a critical nucleus which is characterized by $V_{s}=0$ in the thermodynamic treatment is nothing but a cluster of size $n=1$ from a molecular level perspective. Equation (9), as long as its application is limited to a critical nucleus, is thus quite consistent with the observation that $W$ given by Eq. (6) is zero for $n=1$.

However, Lothe and Pound pointed out an inconsistency of Eq. (9) in that a proper account of the translational and rotational free energies of the cluster is missing in Eq. (9). One cannot account for these effects simply by adding terms containing the translational and the rotational partition functions $Q_{t r}$ and $Q_{r o t}$ of the cluster:

$$
W_{G}-k_{B} T \ln \left(Q_{t r} Q_{r o t}\right) \neq-k_{B} T \ln \frac{\left(V / N_{1}\right) z_{n}}{Y_{v}^{n / N}} .
$$

In fact, when converting $n$ vapor particles into an $n$-sized cluster, no mechanical degrees of freedom are created or destroyed. Thus, the indicated correction would violate what might be called the "conservation of mechanical degrees of freedom." In other words, the left hand side of Eq. (10) depends explicitly on Planck's constant $h$, while the right hand side does not.

Considering a thought process in which a cluster embedded in the bulk liquid is transferred to the vapor phase, Lothe and Pound $[1,27,28]$ argued that the entropy associated with the vibrational translation and rotation of the embedded cluster, with the relative positions of the $n$ particles held fixed, is already included in $W_{G}$, but that such modes of fluctuation become unavailable to the embedded cluster when it is separated from the bulk liquid and placed in the vapor prior to assuming the free translation and rotation corresponding to $Q_{t r}$ and $Q_{r o t}$. The partition function corresponding to those vibrational modes of fluctuation is called the replacement 
partition function and will be denoted by $q_{\text {rep }}$, since these modes are replaced by the free translation and the free rotation in the vapor phase. Note that the vibrational translation and rotation of the embedded cluster requires six mechanical degrees of freedom. Thus, upon division of $Q_{t r} Q_{r o t}$ by $q_{\text {rep }}$, which corresponds to turning off the vibrational translation and rotation prior to activating the free translation and rotation, the superfluous dependence on $h$ disappears, thereby restoring the conservation of the mechanical degrees of freedom. The proper procedure to correct Eq. (9), according to Lothe and Pound, is therefore

$$
W_{G}-k_{B} T \ln \frac{Q_{t r} Q_{r o t}}{q_{\text {rep }}}=-k_{B} T \ln \frac{\left(V / N_{1}\right) z_{n}}{Y_{v}^{n / N}},
$$

and hence

$$
c_{n}=\Phi_{L P} \frac{N_{1}}{V} e^{-W_{G} / k_{B} T},
$$

where

$$
\Phi_{L P}=\frac{Q_{t r} Q_{r o t}}{q_{r e p}}
$$

is the so-called Lothe-Pound factor. Approximating $q_{\text {rep }}$ by $e^{s / k_{B}}$, where $s$ is the entropy of a single particle in the bulk liquid, Lothe and Pound estimated the magnitude of $\Phi_{L P}$ to be $10^{17}$ for a water cluster containing about 100 molecules [1]. A correction factor of a similar magnitude was obtained using the value of $q_{\text {rep }}$ estimated for crystal rather than for liquid $[27,28,31]$. We note that the rate of nucleation is proportional to $c_{n}$ of the critical nucleus. Thus, a correction factor this large appeared excessively large to many, and in some cases, destroyed a fairly good agreement between experiments and classical theory prediction on the critical supersaturation, above which the rate of nucleation becomes noticeable. Thus, a serious controversy has developed since the beginning of the Lothe-Pound theory.

For example, Reiss and his co-workers introduced the concept of a so-called stationary cluster to analyze the problem [9-11]. The partition function of this cluster, by definition, is given by

$$
q_{n}^{s t}=\frac{1}{\Lambda^{3 n} n !} \int_{\mathbf{r}^{n} \in V^{s t}}^{1} d \mathbf{r}^{n} e^{-U_{n} / k_{B} T},
$$

where the $n$ particles are all confined to some volume $V^{s t}$, which in turn is held fixed in space. Then, they assumed that $W_{G}$ is the reversible work of creating this stationary cluster from $n$ vapor particles:

$$
W_{G}=-k_{B} T \ln \frac{q_{n}^{s t}}{Y_{v}^{n / N}} .
$$

If one accepts this assumption, then the remaining problem is that of converting $q_{n}^{s t}$ into $\left(V / N_{1}\right) z_{n}$ by multiplying the former by some appropriate factor $\Phi_{R}$, which then replaces $\Phi_{L P}$ in Eq. (12). This mapping problem has been discussed intensely by Reiss and his co-workers [9-12]. For this purpose, they deactivated the translational motion resulting from the fluctuation of the position of the center of mass of the stationary cluster prior to activating the free translation corresponding to $Q_{t r}$. Since the rotational motion of the $n$ particles inside the container $V^{s t}$ is essentially free rotation, no explicit account needs to be taken for the rotational partition function. When $V^{s t}$ was given a value ranging from $V_{s}$ to several times $V_{s}$, their mapping procedure led to a value of $\Phi_{R}$ ranging from $10^{6}$ to $10^{3}$ for a water cluster consisting of 100 molecules [11]. However, some of the configurations generated as a result of the center of mass motion of the stationary cluster are equivalent to those occurring in $z_{n}$ due to deformation of the cluster with its center of mass fixed in space $[28,42]$. Clearly, such configurations should not be removed from $q_{n}^{s t}$. Nishioka et al. estimated the effect of retaining such configurations in $q_{n}^{s t}$ during the deactivation process [42]. In a more recent work [12], Reiss and his coworkers developed an ingenious method which accounts for the same effect, but in a more rigorous manner. The resulting value of $\Phi_{R}$ is of the order of $10^{4}$ divided by the supersaturation ratio [12].

One might expect that the large discrepancy between $\Phi_{L P}$ and $\Phi_{R}$ should allow for a satisfactory resolution of the disagreement by means of experiments. However, the lack of a proper account of the translational and rotational free energies is not the only source of error in classical theory. In fact, a small deviation of $\sigma$ from $\sigma_{\infty}$ can offset the correction factor to a considerable degree. To see this, let us first rewrite Eq. (8) as

$$
W_{G}=\frac{16 \pi \sigma^{3}}{3\left(p_{l}-p_{v}\right)^{2}},
$$

where we used the Laplace equation

$$
p_{l}-p_{v}=\frac{2 \sigma}{R_{s}},
$$

in which $R_{s}$ is the radius of the surface of tension. As mentioned earlier, one replaces $\sigma$ by $\sigma_{\infty}$ in classical theory, thus obtaining the classical approximation for $W_{G}$,

$$
W_{c l}=\frac{16 \pi \sigma_{\infty}^{3}}{3\left(p_{l}-p_{v}\right)^{2}},
$$

from which we find

$$
\frac{W_{G}}{W_{c l}}=\left(\frac{\sigma}{\sigma_{\infty}}\right)^{3} .
$$

Assuming some reasonable value of $W_{c l} \approx 50 k_{B} T$ and setting $\sigma / \sigma_{\infty}=1.15$ for the sake of argument, we see that there is a reduction in the predicted number density of the critical nuclei by a factor of about $10^{11}$ when $W_{G}$ instead of $W_{c l}$ is used for $W$ in Eq. (5), thereby canceling to a large extent the enormous correction factor $\Phi_{L P}$ put forward by Lothe and Pound.

We note that (15) is not the only relationship between $W_{G}$ and a partition function that has been advanced in place of Eq. (11). For example, Ref. [26] assumed that $W_{G}$ represents the reversible work to create, from the constant density profile representing a uniform vapor, the inhomogeneous density profile which is fixed in space and corresponding to the saddle point of the "Euclidean action" in the functional inte- 
gral representation of the partition function of the inhomogeneous vapor phase. The thought process outlined by Lothe and Pound that led them to Eq. (11) makes it clear, however, that any such assumed relation between $W_{G}$ and a partition function already implies a definite statement regarding the modes of fluctuation contributing to such thermodynamic quantities as $p_{l}-p_{v}$ and $\sigma$. Here lies the essence of the LothePound problem: it is the validity of the assumed relationship that any theory of the replacement partition function must first address before considering the subsequent "mapping problem."

\section{GIBBS'S PERSPECTIVE}

In order to develop a theory of the replacement partition function, it is useful to review the thought process Gibbs devised to interpret Eq. (8) on more physical grounds $[3,36,43]$. Briefly, one starts from a composite system consisting of the bulk vapor phase at $\left(T, p_{v}\right)$ and the bulk liquid phase at $\left(T, p_{l}\right)$ both having the same value of the chemical potential. The pressure difference $p_{l}-p_{v}$ must be maintained by a reversible work source. In the first stage, $\langle m\rangle_{l}=\rho_{l} V_{s}$ particles from the vapor are transferred to the bulk liquid so that the volume of the latter increases by $V_{s}$, where $\rho_{l}$ is the particle number density of the bulk liquid and $\langle\cdots\rangle_{l}$ denotes the thermal average taken with respect to the isothermalisobaric ensemble representing the bulk liquid. During this first stage, the reversible work source receives work $V_{s}\left(p_{l}\right.$ $\left.-p_{v}\right)$. In the second stage, we define a cluster embedded in the bulk liquid phase by taking a spherical region of volume $V_{s}$, which on average will contain $\langle m\rangle_{l}=\rho_{l} V_{s}$ particles. No reversible work needs to be expended for this process. In the third stage, the cluster is extruded into the vapor phase through an aperture, which opens and then closes as the cluster passes through it, thereby creating the vapor-liquid interface. In addition to the force required to push the wall separating the two phases toward the liquid phase side, the reversible work source must also exert a force along the line of contact between the cluster surface, defined by the surface of tension, and the edge of the aperture in order to counterbalance the surface tension. The total work done in the third stage is $\sigma A_{s}$.

During the extrusion of the cluster, it loses the interaction with the bulk liquid. At the same time, it must acquire $\langle n\rangle$ $-\langle m\rangle_{l}$ particles from the vapor phase so that the entirety of Gibbs's thought process amounts to creating an $\langle n\rangle$-sized cluster from $\langle n\rangle$ vapor particles, where $\langle\cdots\rangle$ denotes the thermal average taken with respect to the statistical ensemble representing the vapor phase. The difference $\langle n\rangle-\langle m\rangle_{l}$ is the surface excess density multiplied by $A_{s}$. The reversible work required to carry out the adsorption process and the subsequent structural change is then identified with $\sigma A_{s}$ in the Gibbs theory of the interface.

In the case of vapor to liquid phase nucleation far from the spinodal, the molecular content $n$ of a cluster changes much more slowly compared to the characteristic time scale for the cluster to attain internal equilibrium [37]. This is why $n$ appears as an independent variable in Eq. (1), for example.
In any case, we thus specify the molecular content of a critical nucleus by $n$ instead of $\langle n\rangle$, and hence regard Gibbs's thought process as producing an $n$-sized cluster from $n$ vapor particles. However, the similar argument will not hold for the embedded cluster and the thermal averaging appearing in $\langle m\rangle_{l}$ cannot be omitted.

In Gibbs's thought process, no explicit account is taken of the translational and rotational degrees of freedom of the cluster, either in the bulk liquid or in the vapor. In what follows, we shall consider this aspect explicitly during the extrusion process. To avoid the difficulty of defining the mechanical degrees of freedom of an embedded cluster of size $\langle m\rangle_{l}$, we will consider an $m$-sized cluster instead. The extrusion of the $\langle m\rangle_{l}$-sized cluster is then understood as a result of thermal averaging for various values of $m$ taken with respect to the isothermal-isobaric ensemble representing the bulk liquid. Exactly how this averaging comes about will become clear in Sec. V. The process may be carried out in the following steps.

(1) Turn off the translational and rotational motion of the $m$ sized cluster in bulk liquid with respect to the spherical volume $V_{s}$, which is fixed in space.

(2) Extrude the cluster without activating the translational or the rotational motion with respect to the spherical volume $V_{s}$, which upon the completion of the extrusion process is held fixed in the vapor phase. The interaction between the cluster and the surrounding liquid will be lost.

(3) Activate the free translation within $V$ and the free rotation of the cluster.

(4) Transfer $n-m$ particles from the vapor to the cluster and allow the cluster to go through the structural relaxation.

(5) Replace the free translation within $V$ by that in $V / N_{1}$.

By giving the free translation and rotation of the cluster prior to step 4, we avoid the difficulty of controlling the translational and rotational degrees of freedom during the adsorption of the particles.

Once a certain relationship is assumed between the reversible work required to carry out each of these steps on the one hand and such quantities as $q_{\text {rep }}$ and $\sigma A_{s}$ on the other, we can derive an expression for the correction factor. We shall postpone the actual formulation until Sec. V and analyze the original Lothe-Pound and Reiss prescriptions for $q_{\text {rep }}$ in the next section.

\section{IMPLICATIONS OF THE LOTHE-POUND AND REISS PRESCRIPTIONS FOR $q_{\text {rep }}$}

In both of these theories, the distinction between $n$ and $m$ is ignored, and hence they tacitly employ the equimolar dividing surface in place of the surface of tension. Strictly speaking, therefore, some of the equations in Sec. II must be modified so that $\sigma$ can be interpreted as the surface free energy defined with respect to the former dividing surface [43]. Even with this dividing surface, however, the distinction between $n$ and $m$ must be made in dealing with multicomponent systems, since the surface excess densities cannot be made to vanish simultaneously for all species. In analyzing these theories, therefore, we shall assume that the surface of tension is the dividing surface being used and then regard 
the relation $n=m$ as an approximation rather than a statement on the choice of the dividing surface.

The Lothe-Pound prescription for $q_{\text {rep }}$ can best be understood by dividing Gibbs's extrusion process into the following steps:

(1) Turn off the translational and rotational motion of the $n$-sized cluster in the bulk liquid with respect to the spherical volume $V_{s}$, which is fixed in space.

(2) Extrude the cluster without activating the translational or the rotational motion with respect to the spherical volume $V_{s}$. As before, $V_{s}$ is held fixed in the vapor phase upon the completion of the extrusion process.

(3) Without activating the translational or rotational motion, allow the cluster to go through the structural relaxation.

(4) Activate the free translation within $V / N_{1}$ and the free rotation of the cluster.

These steps differ from those considered in Ref. [2], but allow us to illustrate the essential point. In the Lothe-Pound theory, $q_{\text {rep }}$ is understood as the partition function associated with the translational and rotational motion that is turned off in step 1. This in turn implies that the reversible work associated with steps 2 and 3 is assumed to be $\sigma A_{s}$.

The extrusion process can also be divided into steps so as to involve a stationary cluster.

(1) Turn off the translational and rotational motion of the $n$-sized cluster in the bulk liquid with respect to the spherical volume $V_{s}$, which is fixed in space.

(2) Extrude the cluster without activating the translational or the rotational motion with respect to the spherical volume $V_{s}$. As before, $V_{s}$ is held fixed in the vapor phase upon the completion of the extrusion process.

(3) Allow the cluster to go through structural relaxation and at the same time activate the constrained translational and rotational motion of the cluster only so far as the constraint $\mathbf{r}^{n} \in V^{s t}$ is satisfied, where $V^{s t}$ is also held fixed in the vapor phase and contains $V_{s}$ in it.

(4) Turn off that part of the constrained translational and rotational motion resulting from linear translation and rotation of the cluster within $V^{s t}$ with relative positions of the $n$ particles fixed.

(5) Activate the free translation within $V / N_{1}$ and the free rotation of the cluster in vapor.

We note that the cluster obtained at the end of step 3 is exactly the stationary cluster. Insofar as the rotation described in step 4 is essentially the free rotation, the associated rotational partition function cancels out its counterpart in step 5. Thus, we see that the theory of Reiss and his co-workers [12] is equivalent to interpreting $q_{\text {rep }}$ as the partition function associated with the translational and rotational motion that is turned off in step 4. It follows that the Reiss prescription for $q_{\text {rep }}$, as encoded by Eq. (15), amounts to identifying $\sigma A_{s}$ with the reversible work required to carry out steps 1 through 3.

We can gain some qualitative understanding of the principal difference between the Lothe-Pound and the Reiss prescriptions by considering their implications for the usual classical theory, in which $\sigma$ is replaced by $\sigma_{\infty}$. In order to obtain an $n$-sized cluster having the surface tension $\sigma_{\infty}$ over at least a part of its surface, let us take the spherical volume $V_{s}$ in such a way that a part of its surface passes through the transition layer of the macroscopic vapor-liquid interface. The extent over which the embedded cluster can freely translate and rotate will be different from that in the bulk liquid because of a change in the interaction between the cluster and the surrounding molecules. Lothe and his co-workers refer to this situation as the "release of correlation." (See Refs. $[5,8,44]$ for example.) Thus, as they pointed out, the reversible work $\sigma_{\infty} A_{s}$ associated with the creation of the surface, and hence $\sigma_{\infty}$ itself, contains some contribution from this release of correlation. In the original Lothe-Pound prescription for $q_{r e p}$, however, it was implicitly assumed that no effect of the release of correlation is reflected in $\sigma_{\infty}$. In the Reiss prescription, in contrast, $\sigma_{\infty}$ is assumed to reflect the effect of a significant release of the translational correlation and almost complete release of the rotational correlation, though the actual extent of this release depends on the choice of $V^{\text {st }}$. The actual effect of the release of correlation reflected in the experimentally determined value of $\sigma_{\infty}$ will be somewhere between these two extremes. Thus, if the size dependence of $\sigma$ can be ignored, these two theories bracket the correction factor needed to bring the usual classical theory in line with the correct formula Eq. (1).

The concept of the release of correlation seems difficult to formulate when one is concerned with $\sigma$ rather than $\sigma_{\infty}$. Given the subtleties originating from this concept, one might be tempted to adopt either of the two prescriptions as defining the surface tension $\sigma$. Proceeding in this manner, one can, in principle, determine the value of $\sigma$ by means of molecular level theories along with the Laplace equation. (More specifically, one would first choose $R_{S}$ and then evaluate $\sigma$ from the free energy change of the cluster as it goes through the relevant steps, whichever prescription one decides to work with. Then, the process could be repeated until the Laplace equation is satisfied by the $R_{s}$ one chose and the resulting value of $\sigma$.) Moreover, the problem of the release of correlation seemingly disappears by virtue of the definition. The value of $\sigma$ so defined will depend on the theory one employs. The predicted equilibrium cluster size distribution $c_{n}$, however, will be independent of the theory employed in this purely statistical mechanical, yet extremely indirect, approach. The point, however, is that for the purpose of rectifying classical theory and then developing a useful phenomenological theory of nucleation, $\sigma$ so defined must still be connected to the experimentally accessible $\sigma_{\infty}$.

\section{FORMALISM FOR THE REPLACEMENT PARTITION FUNCTION}

Motivated by the Lothe-Pound prescription for $q_{\text {rep }}$, we shall develop a statistical mechanical expression of $q_{\text {rep }}$ based on the extrusion process we devised in Sec. III and then evaluate the corresponding correction factor, which we shall continue to refer to as the Lothe-Pound factor and denote by $\Phi_{L P}$ despite the differences between our extrusion process in Sec. III and its counterpart in the Lothe-Pound theory as described in Sec. IV.

The isothermal-isobaric partition function of the bulk liquid held at constant $\left(T, p_{l}, N\right)$ is given by 


$$
Y\left(T, p_{l}, N\right)=\int \frac{d V}{a} \frac{e^{-p_{l} V / k_{B} T}}{h^{3 N} N !} \int d \mathbf{p}^{N} d \mathbf{r}^{N} e^{-H_{N} / k_{B} T},
$$

where $\mathbf{p}^{N}$ collectively denotes the momentum of each of the $N$ particles and $H_{N}$ is the system Hamiltonian. The constant $a$ arises from the mechanical degrees of freedom of a piston imposing the constant pressure $p_{l}$ and has the dimension of volume. We take a spherical region of volume $V_{s}=4 \pi R_{s}^{3} / 3$ around the origin of the system and partition the phase points embraced by Eq. (20) according to the number $m$ of molecules inside the spherical region:

$$
\begin{aligned}
Y\left(T, p_{l}, N\right)= & \int \frac{d V}{a} e^{-p_{l} V / k_{B} T} \sum_{m=3}^{N} \frac{1}{h^{3(N-m)}(N-m) !} \\
& \times \int_{\mathbf{r}^{N-m} \in V-V_{s}} d \mathbf{p}^{N-m} d \mathbf{r}^{N-m} e^{-H_{N-m} / k_{B} T} \\
& \times \frac{1}{h^{3 m} m !} \int_{\mathbf{r}^{m} \in V_{S}} d \mathbf{p}^{m} d \mathbf{r}^{m} e^{-H_{m} / k_{B} T} e^{-U_{i n t} / k_{B} T},
\end{aligned}
$$

where $U_{\text {int }}$ denotes the interaction potential between the $N$ $-m$ particles outside the sphere and the $m$ particles inside the sphere, and is a function of $\mathbf{r}^{N-m}$ and $\mathbf{r}^{m}$. It is this dependence of $U_{i n t}$ on $\mathbf{r}^{N-m}$ and $\mathbf{r}^{m}$ that leads to the correlation discussed in Sec. IV. In contrast, $H_{m}$ is the sum of the kinetic energy of $m$ particles inside $V_{s}$ and the interaction potential among them, and hence depends only on $\mathbf{p}^{m}$ and $\mathbf{r}^{m}$. Similarly for $H_{N-m}$. We have explicitly excluded terms with $m \leqslant 2$ as needed by the later development. This is a reasonable approximation for liquid phases provided that $\rho_{l} V_{s}$ is not too small.

It is noted that in transforming Eq. (20) to Eq. (21), we simply classified the phase points occurring in the former according to the number $m$ of molecules which happen to be found inside the spherical region of volume $V_{s}$ and no explicit constraint is introduced. In fact, it is straightforward to show that Eqs. (20) and (21) reduce to an identical expression when applied to an ideal gas provided that the terms $m=0,1$, and 2 are retained in the latter.

Our choice of the spherical shape for $V_{s}$ is consistent with the Gibbs extrusion process discussed in Sec. III. It might seem equally reasonable to choose a nonspherical shape for the volume $V_{s}$ depending perhaps on the shape the cluster takes upon extrusion and the subsequent structural relaxation. However, the Gibbs formula for $W_{G}$ remains valid down to vanishingly small size of the critical nucleus if and only if the shape of the critical nucleus, on average, is spherical [36]. Thus, the very meaning of such quantities as the surface tension may become illusory when referring to nonspherical clusters, and hence it seems hardly worthwhile to try to formulate the Lothe-Pound theory in such a manner as to include nonspherical clusters. For clusters of truncated and shifted Lennard-Jones particles, which we study in Sec. VI, the assumption of spherical clusters is reasonable.

The last integral in Eq. (21), along with the coefficient $1 / h^{3 m} m$ !, may be regarded as the partition function $\xi_{m}$ of a cluster, which consists of $m$ particles, all confined to $V_{s}$, and is embedded in the liquid phase. We move from a laboratory coordinate system to a body coordinate system centered around the center of mass $\mathbf{R}_{\text {c.m. }}$. of this embedded cluster. It is not necessary to specify the actual choice of this body coordinate system for the subsequent development and various possibility exists. For example, Nishioka et al. [45] defined the body coordinate system by first choosing its orientation at some given instant so that the first and second particles are located on its $x$ axis and $x y$ plane, respectively. Then, the subsequent orientation of the coordinate system is defined so that the angular velocity of the cluster in the body coordinate system is zero at all time. In other words, this body coordinate system "rotates with the cluster."

Denoting by $\mathbf{s}^{m-2}$ and $\mathbf{t}^{m-2}$ the coordinates and the conjugate momenta of the remaining 3m-6 degrees of freedom of the embedded cluster, we arrive at

$$
\begin{aligned}
\xi_{m}= & \frac{1}{h^{3 m-6} m !} \int_{\mathbf{r}^{m} \in V_{s}} d \mathbf{t}^{m-2} d \mathbf{s}^{m-2} e^{-K_{s} / k_{B} T} e^{-U_{m} / k_{B} T} \\
& \times \frac{\zeta_{1} \zeta_{2} \zeta_{3}}{\Lambda_{c . m .}^{3}} \int_{\mathbf{r}^{m} \in V_{s}} d \mathbf{R}_{c . m .} \sin \theta d \theta d \phi d \psi e^{-U_{i n t} / k_{B} T},
\end{aligned}
$$

where $K_{s}$ is the kinetic energy of the $m$ particles excluding those due to rigid translation and rotation of the embedded cluster as a whole, $U_{m}$ is the interaction potential among the $m$ particles, and the Euler angles $(\theta, \phi, \psi)$ specify the orientation of the cluster. Finally,

$$
\Lambda_{c . m .}=\frac{h}{\sqrt{2 \pi M k_{B} T}} \quad \text { and } \quad \zeta_{i}=\frac{\sqrt{2 \pi I_{i} k_{B} T}}{h}
$$

with $M$ and $I_{i}(i=1,2,3)$ denoting the mass of the cluster and its principal moments of inertia, respectively.

The second integral in Eq. (22) may be regarded as the configurational partition function $Z_{c}$ of the embedded cluster due to its translational and rotational degrees of freedom when it is subjected to the external field $U_{\text {int }}$. Thus, we may define the configurational entropy $S_{c}$ associated with these degrees of freedom by means of the equation

$$
\begin{aligned}
Z_{c} & =\int_{\mathbf{r}^{m} \in V_{s}} d \mathbf{R}_{c . m .} \sin \theta d \theta d \phi d \psi e^{-U_{\text {int }} / k_{B} T} \\
& =\delta^{3} e^{-\left\langle U_{\text {int }}\right\rangle_{c} / k_{B} T} e^{S_{c} / k_{B}}
\end{aligned}
$$

where $\langle\cdots\rangle_{c}$ denotes the thermal average taken with the Boltzmann weight $e^{-U_{\text {int }} / k_{B} T}$ while imposing the constraint $\mathbf{r}^{m}$ $\in V_{s}$ that the $m$ particles are confined to the volume $V_{s}$. The quantity $\delta$ is an arbitrary length scale and is introduced here to make explicit the dimensionality of various quantities involved. A natural choice for $\delta$ is to identify it with some characteristic length scale of the system such as the particle diameter. Note that the numerical value of $Z_{c}$, and hence that of $\delta^{3} e^{S_{c} / k_{B}}$, depends only on the functional form of $U_{i n t} / k_{B} T$ and the unit used to measure a length. Once they are specified, the numerical value of $\delta^{3} e^{S_{c} / k_{B}}$ is independent of the choice of $\delta$ as seen from Eq. (24). The equation also indicates that $S_{c} / k_{B}$ is independent of the choice of the unit of 
length since a change in the unit affects both $Z_{c}$ and $\delta^{3}$ in an identical manner. Thus, $q_{\text {rep }}$ and $\Phi_{L P}$ given below are independent of our choice of $\delta$ and the unit of length.

Since the coordinates $\mathbf{r}^{N-m}$ and $\mathbf{s}^{m-2}$ are fixed when evaluating $Z_{c}$, the translational and rotational motion of the embedded cluster is seen to be a vibrational one, just as in the embedded cluster considered by Lothe and Pound. [See the paragraph containing Eq. (11).] Our theory differs from theirs because of the constraint $\mathbf{r}^{m} \in V_{s}$ in ours. We emphasize that $U_{\text {int }}$, and hence $\left\langle U_{i n t}\right\rangle_{c}$ and $S_{c}$, are all functions of $\mathbf{s}^{m-2}$ and $\mathbf{r}^{N-m}$.

Let us first consider extruding a cluster with the fixed internal configuration $\mathbf{s}^{m-2}$ from the bulk liquid to the vapor phase. As we saw in Sec. III, we need to first deactivate the modes of fluctuation associated with the factor

$$
\frac{\zeta_{1} \zeta_{2} \zeta_{3}}{\Lambda_{c . m .}^{3}} \delta^{3} e^{S_{c} / k_{B}}
$$

in $\xi_{m}$. In the course of the time evolution of the bulk liquid phase, many distinct sets of coordinates are generated for the embedded cluster and the surrounding $N-m$ particles. The value of $m$ will also fluctuate. Extrusion of each of the embedded clusters to the vapor phase requires the deactivation of the modes of fluctuations whose contribution to the partition function $\xi_{m}$, and hence to $Y$, is given by Eq. (25) evaluated for the particular set of $\mathbf{r}^{N-m}$ and $\mathbf{s}^{m-2}$. As in the LothePound prescription, we recognize the net effect of this deactivation as the division of $Y$ by $q_{\text {rep }}$. Thus,

$$
\begin{aligned}
\frac{Y\left(T, p_{l}, N\right)}{q_{\text {rep }}}= & \int \frac{d V}{a} e^{-p_{l} V / k_{B} T} \sum_{m} \frac{1}{h^{3(N-m)}(N-m) !} \\
& \times \int_{\mathbf{r}^{N-m} \in V-V_{s}} d \mathbf{p}^{N-m} d \mathbf{r}^{N-m} e^{-H_{N-m} / k_{B} T} \\
& \times \frac{1}{h^{3 m-6} m !} \int_{\mathbf{r}^{m} \in V_{s}} d \mathbf{t}^{m-2} d \mathbf{s}^{m-2} \\
& \times e^{-K_{s} / k_{B} T} e^{-U_{m} / k_{B} T} e^{-\left\langle U_{i n t}\right\rangle_{c} / k_{B} T} .
\end{aligned}
$$

Dividing both sides of Eq. (26) by $Y$ and recognizing the numerator on the right hand side of the resulting equation as $Y$ short of the factor given in Eq. (25) in its integrand, we find

$$
\frac{1}{q_{\text {rep }}}=\left\langle\left(\frac{\zeta_{1} \zeta_{2} \zeta_{3}}{\Lambda_{c . m .}^{3}} \delta^{3} e^{S_{c} / k_{B}}\right)^{-1}\right\rangle,
$$

where, as before, $\langle\cdots\rangle_{l}$ indicates a thermal average taken in the bulk liquid held at constant $\left(T, p_{l}, N\right)$.

Activation of the free translation within the volume $V$ and the free rotation of the extruded cluster, when averaged over all possible values of $m$ and internal configurations $\mathbf{s}^{m-2}$, leads to the factor

$$
Q_{t r} Q_{r o t}=\left\langle\frac{\zeta_{1} \zeta_{2} \zeta_{3}}{\Lambda_{c . m .}^{3}}\right\rangle_{l} 8 \pi^{2} V
$$

Upon the extrusion, the $m$-sized cluster loses its interaction $U_{i n t}$ with the surroundings, acquires $n-m$ particles from the vapor phase, and then undergoes the structural relaxation.
Thus, the translational and the rotational partition functions of the $n$-sized cluster differ from the corresponding quantities given in Eq. (28) for the $m$-sized cluster. Nevertheless, as in the original Lothe-Pound prescription, we assume that the reversible work associated with these processes is included fully in $\sigma$. Replacing the free translation within $V$ by that in $V / N_{1}$, we finally arrive at the expression of the Lothe-Pound factor

$$
\Phi_{L P}=\left\langle\frac{\zeta_{1} \zeta_{2} \zeta_{3}}{\Lambda_{c . m .}^{3}}\right\rangle \frac{8 \pi^{2} V}{N_{1}}\left\langle\left(\frac{\zeta_{1} \zeta_{2} \zeta_{3}}{\Lambda_{c . m .}^{3}} \delta^{3} e^{S_{c} k_{B}}\right)^{-1}\right\rangle_{l} .
$$

We observe that $\Phi_{L P}$ involves two thermally averaged quantities both taken with respect to the isothermal-isobaric ensemble representing the bulk liquid. Thus, $\Phi_{L P}$ expresses the deactivation of the translational and rotational degrees of freedom of the $\langle m\rangle_{l}$-sized cluster in the bulk liquid followed by the activation of the same degrees of freedom of the $\langle m\rangle_{l}$-sized cluster in the vapor phase without the adsorption of excess number of molecules from the vapor phase or the subsequent structural relaxation. In this regard, $\Phi_{L P}$ given in Eq. (29) is quite consistent with Gibbs's original extrusion process. If we were to first figure out the effect on the partition function of an $m$-sized cluster due to turning off and on these degrees of freedom, and then take the average over all possible $m$-sized clusters, the resulting expression for $\Phi_{L P}$ would involve only a single thermally averaged quantity. This latter possibility is not permissible since $-k_{B} T \ln \Phi_{L P}$ should yield the free energy difference between the two states of the cluster, one placed in the vapor phase and the other embedded in the bulk liquid, both with translational and rotational degrees of freedom properly activated.

Perhaps it is worth noting that Eq. (29) also follows from considering the Lothe-Pound extrusion process described in Sec. IV except that the only value of $m$ contributing to the thermal average in Eq. (29) in that case will be $n$.

To estimate $Z_{c}$ by means of computer simulation, we consider an auxiliary partition function

$$
Z=\int d \mathbf{R}_{c . m .} \sin \theta d \theta d \phi d \psi e^{-U_{i n t} / k_{B} T},
$$

in which the constraint $\mathbf{r}^{m} \in V_{s}$ is discarded, and recognize the ratio $Z_{c} / Z$ as the probability that the condition $\mathbf{r}^{m} \in V_{s}$ is satisfied when the embedded cluster is allowed to move around under the influence of the external field $U_{i n t}$ but without the constraint $\mathbf{r}^{m} \in V_{s}$. This probability can be estimated directly by simulation. The auxiliary partition function $Z$ can be evaluated by a standard thermodynamic integration method [46], in which we specify the integration path by

$$
\begin{gathered}
U_{\lambda}=\lambda U_{i n t}+(1-\lambda) U_{H O} \quad(0 \leqslant \lambda \leqslant 1), \\
U_{H O}=U_{0}+\alpha\left(\frac{1}{\delta^{2}}\left|\mathbf{R}_{c . m .}-\mathbf{R}_{c . m .}^{0}\right|^{2}+U_{r o t}\right),
\end{gathered}
$$

and

$$
U_{r o t}=2(1-\cos \theta)+(\phi+\psi)^{2},
$$

where $\alpha$ is a constant having the dimension of energy and $U_{0}$ is a local minimum of $U_{\text {int }}$ realized at $\mathbf{R}_{c . m .}=\mathbf{R}_{c . m \text {. }}^{0}$ with a 
suitable orientation, for which we may arbitrarily assign the Euler angles $\theta=0, \phi=0, \psi=0$ for the sake of carrying out the integration indicated in Eq. (30). For a small angle rotation, $U_{r o t}$ is quadratic in the net rotation angle of the cluster [47], and hence reduces to a harmonic potential. The $1 / \delta^{2}$ factor in Eq. (32) ensures dimensional consistency. The partition function of the reference state $(\lambda=0)$, obtained by replacing $U_{\text {int }}$ in Eq. (30) by $U_{H O}$, can be evaluated analytically, thus yielding

$$
\begin{aligned}
Z= & \delta^{3}\left(\frac{\pi k_{B} T}{\alpha}\right)^{3} \gamma \\
& \times \exp \left[-\frac{1}{k_{B} T}\left(U_{0}+\int_{0}^{1}\left\langle U_{i n t}-U_{H O}\right\rangle_{\lambda} d \lambda\right)\right],
\end{aligned}
$$

where $\langle\cdots\rangle_{\lambda}$ denotes the average taken with Boltzmann's factor $e^{-U_{\lambda} / k_{B} T}$ and

$$
\gamma=\frac{1-e^{-4 \alpha / k_{B} T}}{2 \pi} \int_{0}^{2 \pi} d x \operatorname{erf}\left(\sqrt{\frac{\alpha}{k_{B} T}}(2 \pi-x)\right) .
$$

In principle, the value of the constant $\alpha>0$ is arbitrary, but affects the accuracy of the estimated value of the integral in Eq. (34). We adjusted $\alpha$ so that no single term dominates the summation used to approximate the integral.

\section{RESULTS}

To estimate the magnitude of $\Phi_{L P}$, simulation was carried out for the Lennard-Jones fluids. Thus, we use LennardJones units in what follows. The potential was truncated at radius 2.5 and shifted upward so that it will vanish there. $\delta$ was set to unity. Bulk liquid containing a total of 1600 particles was subjected to isothermal-isobaric Monte Carlo (MC) simulation at three temperature values $0.7,0.8$, and 0.9. At each temperature, four values of the liquid pressure $p_{l}$, i.e., $p_{\text {sat }}, 0.04,0.08$, and 0.16 , were used, where $p_{\text {sat }}$ is the saturation pressure and was determined by means of Gibbs ensemble simulation [48].

At every $0.5 \times 10^{5} \mathrm{MC}$ steps, where one $\mathrm{MC}$ step consists on average of one attempted volume change move and one trial displacement of each particle, the particles were separated into those belonging to the embedded cluster and those of the surroundings, using spherical boundaries of radii $R_{s}=1.8,2$, and 2.5. We note that, for a given intensive state of the metastable vapor, the value of $R_{s}$ is in principle determined uniquely by Eq. (17), in which $\sigma$, however, would have to be evaluated by a separate simulation based on the physical content we assigned to it in Sec. III. The $R_{s}$ values we used here are believed to be of a reasonable magnitude. The resulting values of $\langle m\rangle_{l}$ shown in Figs. 1-3 are in line with typical size of a critical nucleus for this type of model systems.

Each cluster so generated was subjected to rigid MC translation and rotation to find an approximate minimum potential energy configuration and the associated $U_{0}$ value. The optimum choice of the constant $\alpha$ varies from one cluster to another and was found by a few repetitions of trial and error in each case, which produced typical values of $\alpha$ in the range

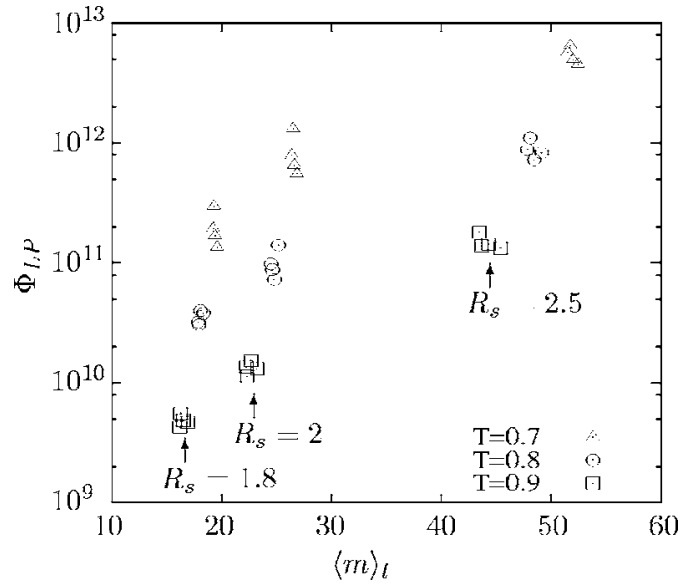

FIG. 1. The Lothe-Pound factor $\Phi_{L P}$ vs the average number $\langle m\rangle_{l}$ of the particles in the embedded cluster taken inside the bulk liquid phase.

of 700-2500. The integral in Eq. (34) was evaluated by means of the trapezoidal rule [49], in which the interval 0 $\leqslant \lambda \leqslant 1$ was divided into 80 equal intervals. The integrand at each $\lambda$ value was estimated using $10^{5}$ MC steps, where each MC step consists on average of one rigid translation and one rigid rotation of the embedded cluster with the surrounding $N-m$ particles held fixed in space. From this simulation, the values of $Z_{c},\left\langle U_{\text {int }}\right\rangle_{c}$, and hence $S_{c}$ were determined for a given configuration specified by $\mathbf{r}^{N-m}$ and $\mathbf{s}^{m-2}$. Then, at each state point and each value of $R_{s}$, the thermal averages appearing in Eq. (29) were estimated from 100 distinct configurations specified by $\mathbf{r}^{N-m}$ and $\mathbf{s}^{m-2}$, where the value of $m$ depends on the instantaneous configuration in bulk liquid at the moment an embedded cluster is identified.

To each value of $p_{l}$, the corresponding values of $p_{v}$ were obtained by equating the chemical potentials of the two phases, which in turn were obtained by integrating the Gibbs-Duhem relation for each phase along an isotherm starting from phase coexistence. The average volume per particle $v_{l}$ of the bulk liquid, which is needed to integrate the Gibbs-Duhem equation, was estimated directly by conduct-

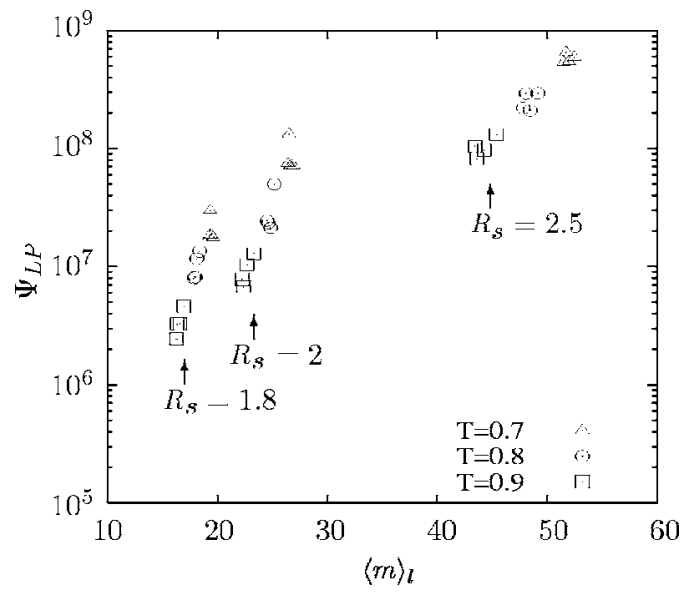

FIG. 2. The part of the Lothe-Pound factor depending only on the properties of the bulk liquid vs $\langle m\rangle_{l} . \Psi_{L P}$ is given in LennardJones units. 


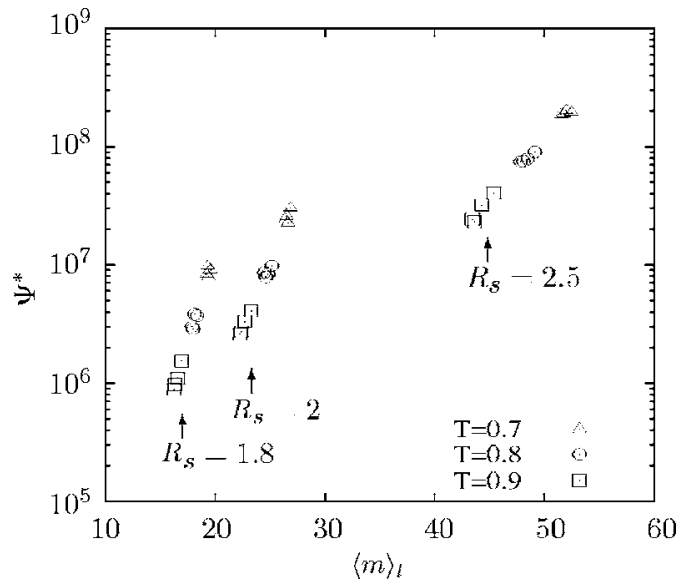

FIG. 3. The same as Fig. 2 except that $\Psi^{*}$ was obtained by discarding the constraint that $\mathbf{r}^{m} \in V_{s} . \Psi^{*}$ is given in Lennard-Jones units.

ing a series of isothermal-isobaric simulations over the pressure range indicated. The corresponding quantity for the vapor phase was estimated from the virial equation of state using up to the second virial coefficient [50]. Once $p_{v}$ was obtained for a given value of $p_{l}$ in this manner, the factor $V / N_{1}$ in Eq. (29) was calculated again from the virial equation of state assuming the vapor phase to consist entirely of the monomers.

The resulting values of $\Phi_{L P}$ are shown in Fig. 1, and seen to depend very sensitively on $T$ and $\langle m\rangle_{l}$, where $\langle m\rangle_{l}$ was calculated as $V_{s} / v_{l}$. The data tend to cluster into groups, each corresponding to given values of $T$ and $R_{S}$ and containing four data points, each representing the value of $\Phi_{L P}$ at a given value of $p_{l}$. No systematic trend was observed with the variation of $p_{l}$ at given $T$ and $R_{s}$. This in part is due to the statistical error in our estimate of $\Phi_{L P}$. In addition, the $p_{l}$ dependence of $q_{\text {rep }}$ and that of $V / N_{1}$ tend to cancel each other.

Our estimate of $\Phi_{L P}$ is somewhat smaller than the original value of $10^{17}$. Recalling that the latter was evaluated for a water cluster containing about 100 molecules using the value of $q_{\text {rep }}$ suitable for crystal nucleation, the values we obtained may be said to be in line with the original estimate by Lothe and Pound. Nevertheless, the sensitive dependence on $T$ and $\langle m\rangle_{l}$ points to the necessity of a detailed calculation of $\Phi_{L P}$ as reported here.

It is of interest to examine more closely the individual factors contributing to $\Phi_{L P}$. For example, we may extract from Eq. (29) the factors depending only on properties of the bulk liquid phase:

$$
\Psi_{L P}=\left\langle\frac{\zeta_{1} \zeta_{2} \zeta_{3}}{\Lambda_{c . m .}^{3}}\right\rangle_{l}\left\langle\left(\frac{\zeta_{1} \zeta_{2} \zeta_{3}}{\Lambda_{c . m .}^{3}} \delta^{3} e^{S_{c} / k_{B}}\right)^{-1}\right\rangle_{l}
$$

The values of $\Psi_{L P}$ is shown in Fig. 2, while Fig. 3 shows the similar quantity given by

$$
\Psi^{*}=\left\langle\frac{\zeta_{1} \zeta_{2} \zeta_{3}}{\Lambda_{c . m .}^{3}}\right\rangle_{l}\left\langle\left(\frac{\zeta_{1} \zeta_{2} \zeta_{3}}{\Lambda_{c . m .}^{3}} \delta^{3} e^{S^{*} / k_{B}}\right)^{-1}\right\rangle_{l},
$$

in which $S^{*}$ is defined by a relation similar to Eq. (24) but discarding the constraint that $\mathbf{r}^{m} \in V_{s}$ :

$$
Z=\delta^{3} e^{-\left\langle U_{\text {int }}\right\rangle / k_{B} T} e^{S^{*} / k_{B}} .
$$

Note that $\Psi_{L P}$ and $\Psi^{*}$ have the dimension of $1 /$ volume, and given in these figures in Lennard-Jones units.

In principle, $\Psi^{*}$ depends on $R_{s}$ and the intensive state of the bulk liquid specified by $T$ and $p_{l}$. However, as seen from Fig. 3, the $T$ and $p_{l}$ dependence of $\Psi^{*}$ manifests itself primarily through the dependence of $\langle m\rangle_{l}$ on $T$ and $p_{l}$. From this, we may in turn infer that, at least in a simple nonassociating system such as the Lennard-Jones fluids, the value of $\Psi^{*}$ is determined primarily by the harsh repulsive part of the interparticle potential and that the slowly varying attractive part of the potential plays a secondary role. $\Psi_{L P}$ is obtained from $\Psi^{*}$ by introducing the factor $Z / Z_{c}$ in the latter. We note that no new temperature or pressure dependence arises by introducing this factor, and hence $\Psi_{L P}$ is also determined primarily by the repulsive part of the potential. The increased statistical uncertainty in $\Psi_{L P}$ as discerned by comparing Figs. 2 and 3 indicates that much of the statistical uncertainty in $\Phi_{L P}$ comes from that in the numerical evaluation of the ratio $Z_{c} / Z$.

By comparing Figs. 2 and 3, we estimate the statistical error incurred by the current numerical procedure for evaluating $Z_{c} / Z$ to be, at most, less than a factor of 10 . Our choice of $R_{s}$ results in reasonable values of $\langle m\rangle_{l}$ and the value of $\Phi_{L P}$ increases by a factor less than $10^{2}$ as $R_{s}$ is increased from 1.8 to 2.5. It is of interest to devise an improved numerical method of evaluating $Z_{c}$. It is also desirable to attempt a rigorous estimate of the $R_{s}$, for which we have already indicated the basic approach, though the required computational effort would be quite demanding. Nevertheless, our current numerical procedure for evaluating $Z_{c}$ and our choice for $R_{s}$ are adequate for estimating the order of magnitude of $\Phi_{L P}$.

In evaluating the effect of the release of correlation on $\sigma_{\infty}$, one must account for the spatial variation of the local density across the vapor-liquid interface. Furthermore, there appears to be no obvious choice for the configuration of the $m$-sized embedded cluster. As a rough approximation, we ignored these issues completely and estimated $q_{\text {rep }}$ after removing all of the surrounding particles whose $z$ coordinates, in the coordinate system whose origin coincides with the center of the spherical region $V_{s}$, are positive without allowing any structural relaxation. The upper half of the embedded cluster was thus exposed to vacuum. This resulted in the decrease of $\Phi_{L P}$ at most by an order of magnitude for all cases we studied.

\section{REMARKS}

The essential difference between the Lothe-Pound and the Reiss prescriptions lies in the assumed statistical mechanical interpretation of the surface tension of a microscopic critical nucleus. One can correctly argue that such a difficulty can be avoided entirely in a purely statistical mechanical approach to nucleation. In fact, there has been a considerable progress in both our understanding of statistical mechanics of nucleation and computational techniques in recent years. Nevertheless, the usefulness of truly molecular level approaches is still limited in many practical applications of nucleation 
theory, and a phenomenological theory of nucleation must take its place, at least for the time being. Such a theory would involve, by design, some macroscopic parameters whose values must be determined by some macroscopic means. Here again, it appears unavoidable that the connection be examined carefully between the macroscopic parameters of the phenomenology and the molecular level concepts. It is hoped that the current work, along with existing theories aimed at understanding the nature of the replacement partition function, contributes toward developing a useful tool in this line of investigation.

\section{ACKNOWLEDGMENTS}

I am grateful for a discussion with Kazumi Nishioka on the Lothe-Pound theory during his brief visit to Caltech. Without his careful exposition, subtleties of the theory would have escaped me entirely. This work was supported by the seed grant and the startup fund provided through the Ohio State University. A part of the computation reported here was made possible by a resource grant from the Ohio Supercomputer Center.
[1] J. Lothe and G. M. Pound, J. Chem. Phys. 36, 2080 (1962).

[2] J. Feder, K. C. Russell, J. Lothe, and G. M. Pound, Adv. Phys. 15, 111 (1966).

[3] W. J. Dunning, in Nucleation, edited by A. C. Zettlemoyer (Marcel Dekker, New York, 1969), pp. 1-67.

[4] J. Lothe and G. M. Pound, in Nucleation (Ref. [3]), pp. 109149.

[5] K. Nishioka and G. M. Pound, Surf. Colloid Sci. 8, 297 (1976)

[6] H. Reiss, Adv. Colloid Interface Sci. 7, 1 (1977).

[7] R. Kikuchi, Adv. Colloid Interface Sci. 7, 67 (1977).

[8] K. Nishioka and G. M. Pound, Adv. Colloid Interface Sci. 7, 205 (1977)

[9] H. Reiss and J. L. Katz, J. Chem. Phys. 46, 2496 (1967).

[10] H. Reiss and J. L. Katz, J. Chem. Phys. 47, 2202 (1967).

[11] H. Reiss, J. L. Katz, and E. R. Cohen, J. Chem. Phys. 48, 5553 (1968).

[12] H. Reiss, W. K. Kegel, and J. L. Katz, Phys. Rev. Lett. 78, 4506 (1997).

[13] R. C. Tolman, J. Chem. Phys. 16, 758 (1948).

[14] R. C. Tolman, J. Chem. Phys. 17, 118 (1949).

[15] R. C. Tolman, J. Chem. Phys. 17, 333 (1949).

[16] F. O. Koenig, J. Chem. Phys. 18, 449 (1950).

[17] H. Reiss, A. Tabazadeh, and J. Talbot, J. Chem. Phys. 92, 1266 (1990).

[18] J. S. van Duijneveldt and D. Frenkel, J. Chem. Phys. 96, 4655 (1992).

[19] I. Kusaka, Z.-G. Wang, and J. H. Seinfeld, J. Chem. Phys. 108, 3416 (1998).

[20] B. Chen and J. I. Siepmann, J. Chem. Phys. 115, 10903 (2001).

[21] B. Chen, J. I. Siepmann, and M. L. Klein, J. Am. Chem. Soc. 125, 3113 (2003).

[22] D. W. Oxtoby and R. Evans, J. Chem. Phys. 89, 7521 (1988).

[23] J. Barrett, J. Chem. Phys. 107, 7989 (1997).

[24] D. Reguera and H. Reiss, J. Chem. Phys. 120, 2558 (2004).

[25] V. Talanquer and D. W. Oxtoby, J. Chem. Phys. 100, 5190 (1994).

[26] Y. Drossinos and P. G. Kevrekidis, Phys. Rev. E 67, 026127
(2003).

[27] J. Lothe and G. M. Pound, J. Chem. Phys. 45, 630 (1966).

[28] J. Lothe and G. M. Pound, J. Chem. Phys. 48, 1849 (1968).

[29] J. Lothe and G. M. Pound, Phys. Rev. 182, 339 (1969).

[30] K. Nishioka, R. Shawyer, A. Bienenstock, and G. M. Pound, J. Chem. Phys. 55, 5082 (1971).

[31] K. Nishioka and G. M. Pound, Acta Metall. 22, 1015 (1974).

[32] F. F. Abraham and J. V. Dave, J. Chem. Phys. 55, 1587 (1971).

[33] F. F. Abraham and J. V. Dave, J. Chem. Phys. 55, 4817 (1971).

[34] W. G. Hoover, A. C. Hindmarsh, and B. L. Holian, J. Chem. Phys. 57, 1980 (1972).

[35] F. F. Abraham and R. N. Kortzeborn, J. Chem. Phys. 57, 1985 (1972).

[36] J. W. Gibbs, The Scientific Papers of J. Willard Gibbs: Vol. 1, Thermodynamics (Ox Bow Press, Woodbridge, CT, 1993), pp. 252-258.

[37] K. Nishioka and I. Kusaka, J. Chem. Phys. 96, 5370 (1992).

[38] P. G. Debenedetti and H. Reiss, J. Chem. Phys. 108, 5498 (1998).

[39] I. Kusaka, J. Chem. Phys. 111, 3769 (1999).

[40] P. G. Debenedetti and H. Reiss, J. Chem. Phys. 111, 3771 (1999).

[41] K. Nishioka and A. Mori, J. Chem. Phys. 97, 6687 (1992).

[42] K. Nishioka, G. M. Pound, J. Lothe, and J. P. Hirth, J. Appl. Phys. 42, 3900 (1971).

[43] K. Nishioka, Phys. Rev. A 36, 4845 (1987).

[44] K. Nishioka, J. Jpn. Assoc. Cryst. Growth 5, 1 (1978).

[45] K. Nishioka, G. M. Pound, and F. F. Abraham, Phys. Rev. A 1, 1542 (1970).

[46] M. P. Allen and D. J. Tildesley, Computer Simulation of Liquids (Oxford University Press, New York, 1987).

[47] H. Goldstein, Classical Mechanics, 2nd ed. (Addison-Wesley, Reading, MA, 1980).

[48] A. Z. Panagiotopoulos, Mol. Phys. 61, 813 (1987).

[49] W. H. Press, S. A. Teukolsky, W. T. Vetterling, and B. P. Flannery, Numerical Recepies (Cambridge University Press, Cambridge, U.K., 1992).

[50] J.-P. Hansen and I. R. McDonald, Theory of Simple Liquids, 2nd ed. (Academic Press, San Diego, 1990). 